\title{
Cryogenic telescope on the Infrared Astronomical Satellite (IRAS)
}

\author{
F. J. Low \\ University of Arizona \\ Steward Observatory \\ Tucson, Arizona 85721
}

\section{A. Beichman}

Jet Propulsion Laboratory

California Institute of Technology

MS 264-338

Pasadena, California 91109

F. C. Gillett

Kitt Peak National Observatory

Tucson, Arizona 85726

J. R. Houck

Cornell University

Center for Radiophysics

and Space Research

220 Space Science Building

Ithaca, New York 14853

\section{G. Neugebauer}

California Institute of Technology

Department of Physics

Pasadena, California 91125

\section{E. Langford}

Jet Propulsion Laboratory

California Institute of Technology

Pasadena, California 91109

\section{R. G. Walker}

Jamieson Science and Engineering, Inc.

Palo Alto, California 94306

\section{R. H. White}

Jet Propulsion Laboratory

California Institute of Technology

Pasadena, California 91109

\begin{abstract}
The Infrared Astronomical Satellite (IRAS)* has completed an unbiased all-sky survey at wavelengths from 10 to $100 \mu \mathrm{m}$. The design and performance of the focal plane array is described with emphasis on in-orbit measurements of the sensitivity and stability. In the four broad spectral bands centered at $12,25,60$, and $100 \mu \mathrm{m}$, the system noise equivalent flux density (NEFD) values are, in $\mathrm{Jy} / \mathrm{Hz}^{1 / 2}, 0.03,0.025,0.046$, and 0.21 , respectively (Jansky $=10^{-26} \mathrm{~W} / \mathrm{m}^{2} / \mathrm{Hz}$ ). For point sources, a single scan at the survey rate of $3.8 \mathrm{arcmin} / \mathrm{s}$ yields limiting flux densities at the 3-sigma confidence level of $0.36,0.30,0.39$, and $1.2 \mathrm{Jy}$. The dc stability of the junction field effect transistor (JFET) amplifiers and the excellent off-axis rejection of the telescope permit total flux measurements of extended infrared emission at levels below $10^{6} \mathrm{Jy} / \mathrm{sr}$. Response of the extrinsic silicon and germanium photodetectors to ionizing radiation is described.
\end{abstract}

Keywords: infrared optics; infrared astronomy; cryogenic telescope; all-sky survey; ionizing radiation.

Optical Engineering 23/2), 122-127 (March/April 1984).

\section{CONTENTS}

1. Introduction

2. Scientific objectives of IRAS and conceptual design of the focal plane

3. Instrument design

3.1. General design

3.2. Design of the focal plane assembly

3.3. Design of the electronic data system

3.4. Performance of the detection system

3.5. Ionization radiation effects

4. Acknowledgments

5. References

Invited Paper IR-104 received Sept. 12, 1983; revised manuscript received Oct. 7, 1983 accepted for publication Jan. 9, 1984; received by Managing Editor Jan. 11, 1984. This paper is a revision of Paper 430-42 which was presented at the SPIE conference on Infrared Technology IX, Aug. 23-25, 1983, San Diego, CA. The paper presented there appears (unrefereed) in SPIE Proceedings Vol. 430

๑) 1984 Society of Photo-Optical Instrumentation Engineers.

\section{INTRODUCTION}

In June 1975, scientists and engineers from the United States, The Netherlands, and the United Kingdom formed a team to evaluate the feasibility of performing an all-sky survey at infrared wavelengths from 10 to $100 \mu \mathrm{m}$ using a cryogenically cooled space-borne telescope. The mission, which was approved late in 1975, would build upon experience gained from the Astronomical Netherlands Satellite (ANS) and on the advanced infrared detector and cooled telescope technologies demonstrated by the series of suborbital rocket flights carried out by workers at Cornell University, ${ }^{1-3}$ the Naval Research Laboratory, ${ }^{4,5}$ and the Air Force Geophysical Laboratory. ${ }^{6}$ The scientific requirements that led to the design of the survey focal plane are discussed below. The prime contractor chosen by NASA was Ball

\footnotetext{
* The Infrared Astronomical Satellite was developed and operated by the Netherlands Agency for Aerospace Programs (NIVR), the U.S. National Aeronautics and Space Administration (NASA), and the U.K. Science and Engineering Research Council Administ
} 
Aerospace Systems Division (BASD), with responsibility for the focal plane assembly given to Rockwell International.

The first version of the Infrared Astronomical Satellite (IRAS) focal plane array was delivered to NASA's Ames Research Center early in 1981 for testing prior to integration into the telescope system. It became apparent at that time that the noise level defining the noise equivalent power (NEP) of the array was that of the metal oxide surface field effect transistor (MOSFET) preamplifiers, considerably higher than the Johnson noise of the load resistors and too high to meet the scientific objectives. Also, the offset voltage of the MOSFETs was unpredictable from one cool-down to another, leading to poor dc stability. Further, the susceptibility of MOSFETs to static discharge damage constituted an unacceptable risk to the focal plane.

The array was transferred to the Jet Propulsion Laboratory (JPL) in mid-1981, where it was retrofitted with self-heating junction field effect transistor (JFET) preamps produced by Infrared Laboratories and new detectors for the $100 \mu \mathrm{m}$ band from Ge:Ga material processed by Cornell University. The new array was integrated into the telescope system early in 1982 after extensive testing at JPL.

This paper discusses the design and performance of the IRAS telescope and focal plane, which was launched on January 25, 1983, and performed satisfactorily in orbit until November 22, 1983, when its supply of liquid helium coolant was exhausted.

\section{SCIENTIFIC OBJECTIVES OF IRAS AND CONCEPTUAL DESIGN OF THE FOCAL PLANE}

A number of important scientific objectives had to be considered in the design of the IRAS focal plane. ${ }^{7}$ From a scientific point of view it was desirable to survey the sky over a broad spectral range in a number of contiguous bands at high sensitivity. Concerns about the availability of high sensitivity detectors, high quality filter and field lens materials, as well as the technical complexity of the assembly resulted in the adoption of the four wavelength bands from 12 to 100 $\mu \mathrm{m}$. The more complex issues of the detailed focal plane layout, field aperture size, and shape were decided on the basis of the several rather specific requirements for the principal goal of the mission-an unbiased all-sky survey.

An all-sky survey is scientifically useful only if it is both complete and reliable. A complete catalog is one in which all of the sources in the sky down to some limiting flux level are included. In practice it is impossible to ensure that every source is included without exception. A statistical completeness requirement of $98 \%$ was placed on the system. A reliable catalog is one that does not contain any falsenonexistent-sources. The statistical requirement on the catalog is that it be $99.8 \%$ reliable. A higher requirement was set on reliability because false sources would probably have rather interesting properties, such as peculiar colors or rapidly varying intensity. If such sources were present in the catalog in large numbers, then considerable time would be required to separate them from the genuinely interesting discoveries. The above requirements were waived in the area immediately surrounding the galactic plane because of its high source density.

Translating the completeness and reliability requirements into hardware specifications was a challenging process. We consider now the various features of the focal plane to see how they contributed to the dual requirements of completeness and reliability. As can be seen from the layout shown in Fig. 3, each color band is represented by two double rows of detectors. Each double row is called a module and contains either seven or eight detectors. Each module is approximately $1 / 2$ degree long in the cross-scan direction as projected on the sky. The in-scan width of the apertures was set at several times the diffraction size for the module's central wavelength. The cross-scan length of the apertures in each band was determined by the expected source density on the sky and the requirement that false sources not be generated by the accidental combination of two or more real sources. Theoretical models of the expected source density as a function of flux level and position on the sky were developed to support this activity. The edges of two adjacent apertures overlap sufficiently to ensure that a source cannot pass undetected between them. Field lenses are used behind the apertures to reimage the exit pupil of the telescope on the entrance aperture in front of each detector. In this way the photometric response of the individual apertures is smoothed, and smaller detectors could be used. The design of a single module is optimized for completeness while at the same time avoiding confusion.

The second module in each color band is included for redundancy and to reduce the number of false sources. A large number of effects can produce source-like pulses in the output data stream, including residual tails from deglitched charged particle events, contamination from the satellite, and sightings of nearby space debris. A true astronomical source should produce one or two output deflections as its image passes over the first module of a given color band; a few seconds later it should produce a second deflection(s) as its image passes over the second module. The time delay between these detections depends on the focal plane geometry, the telescope plate scale, and the scan rate. All of these factors are known at the time of the data reduction and can be used to confirm real sources and reject false ones. Because the apertures in the second module are shifted in the cross-scan direction relative to the apertures in the first module, there is an improvement in the determination of a source's cross-scan position. An increase in reliability at the expense of some completeness is achieved by rescanning the same area of sky on the next orbit (103 min later). A further increase in reliability is gained by repeating the entire process a week to ten days later. The prelaunch models indicated that the combined goals of completeness and reliability could be achieved using the focal plane and procedures described above. The flight data indicate that these goals are being met over all of the sky with the exception of the vicinity of the galactic plane.

As the result of balloon observations, ${ }^{8}$ it was realized early in the design phase that the sky, especially near the galactic plane, would contain complex extended emission in the far-infrared and that this would require a stable dc-coupled readout of the infrared detectors. Although there is no absolute intensity reference, the dc stability of the detectors was designed to permit total flux mapping.

\section{INSTRUMENT DESIGN}

\subsection{General design}

The design of the IRAS system as a whole, including the superfluid helium cooling system developed by BASD, is described elsewhere. ${ }^{9}$ The satellite configuration shown in Fig. 1 and the IRAS orbital geometry, Fig. 2, are shown for reference.

\subsection{Design of the focal plane assembly}

The focal plane assembly (FPA) contains the infrared and visible detectors and associated field optics. It comprises 62 infrared channels and eight visible channels. The infrared channels are divided into eight modules, two for each color band. Each module contains either seven or eight detectors. Figure 3 shows the layout of the FPA. Infrared sources scan across the FPA parallel to the $Y$ axis in all observational modes. Figure 4 shows a cutaway of a single channel of a FPA module. The infrared radiation passes through the spectral filters and is focused onto the detector element by the field lens. The photoconductive detector element responds to infrared radiation by altering its electrical resistance. A pair of junction field effect transistors (JFET) amplifies the photocurrent from each detector. The visible wavelength channels are of a similar construction, using silicon diode detectors and a single metal oxide surface field effect transistor (MOSFET) preamplifier per detector. The visible wavelength detectors are placed in a "V" arrangement to provide two-axis spacecraft attitude information from star crossings.

Table I lists the relevant parameters of the design. Figure 5 shows the relative spectral response of the four infrared filter/detector combinations that define the wavelength response of the system.

\subsection{Design of the electronic data system}

An important aspect of the IRAS design is the analog and digital data system that amplifies and processes the photocurrent provided 


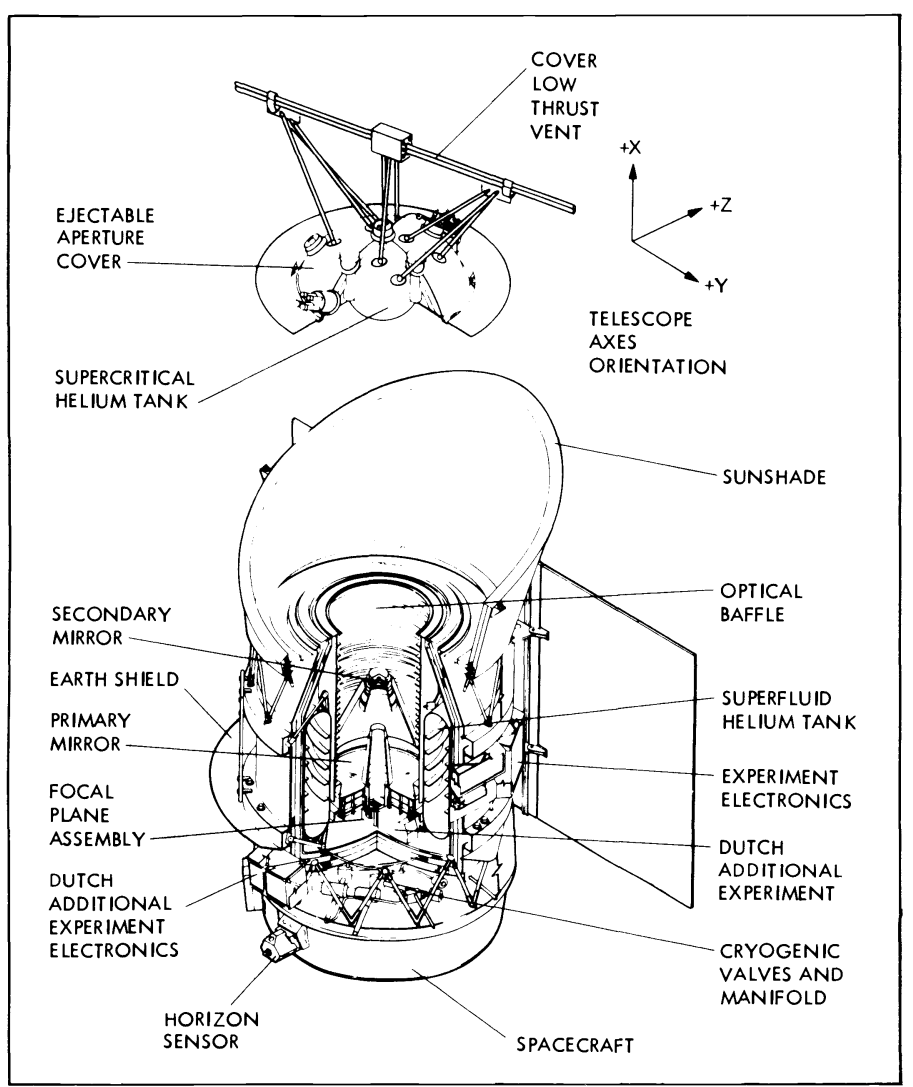

Fig. 1. Satellite configuration.

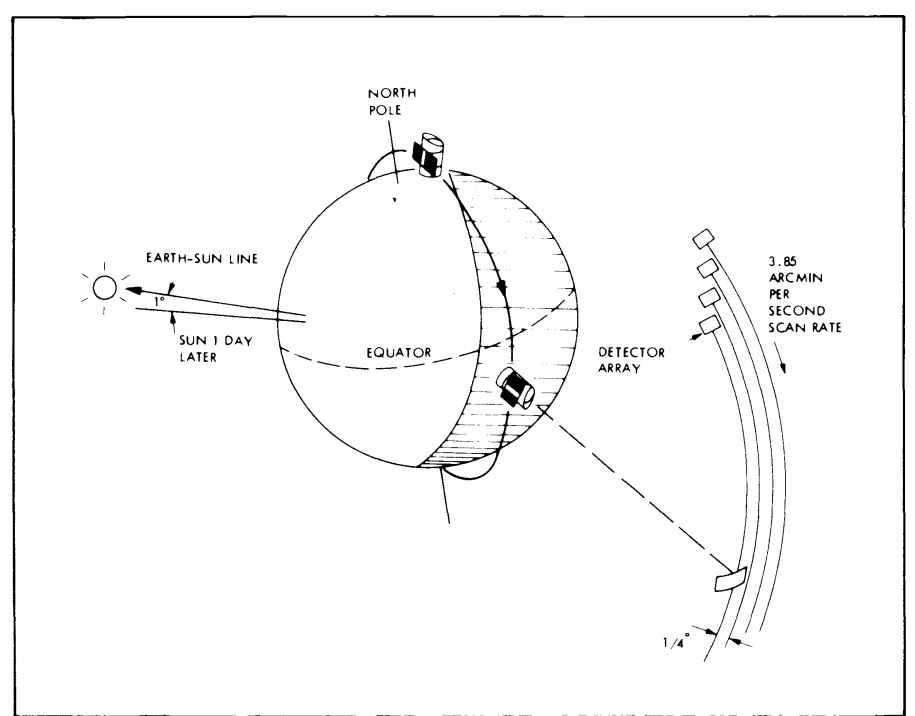

Fig. 2. IRAS orbit geometry.

by the detectors. A key element in this design is the self-heated, cryogenically cooled, JFET preamplifier described by Low. ${ }^{10}$ The flow of data through amplification, an on-board deglitcher to reduce the effect of ionizing particle events, analog filtering, and, finally, digitization is depicted in Fig. 6.

\subsection{Performance of the detection system}

The IRAS focal plane and its related systems were tested prior to launch. Table II summarizes the results of these tests and their analyses. It is, of course, the in-flight performance of the various

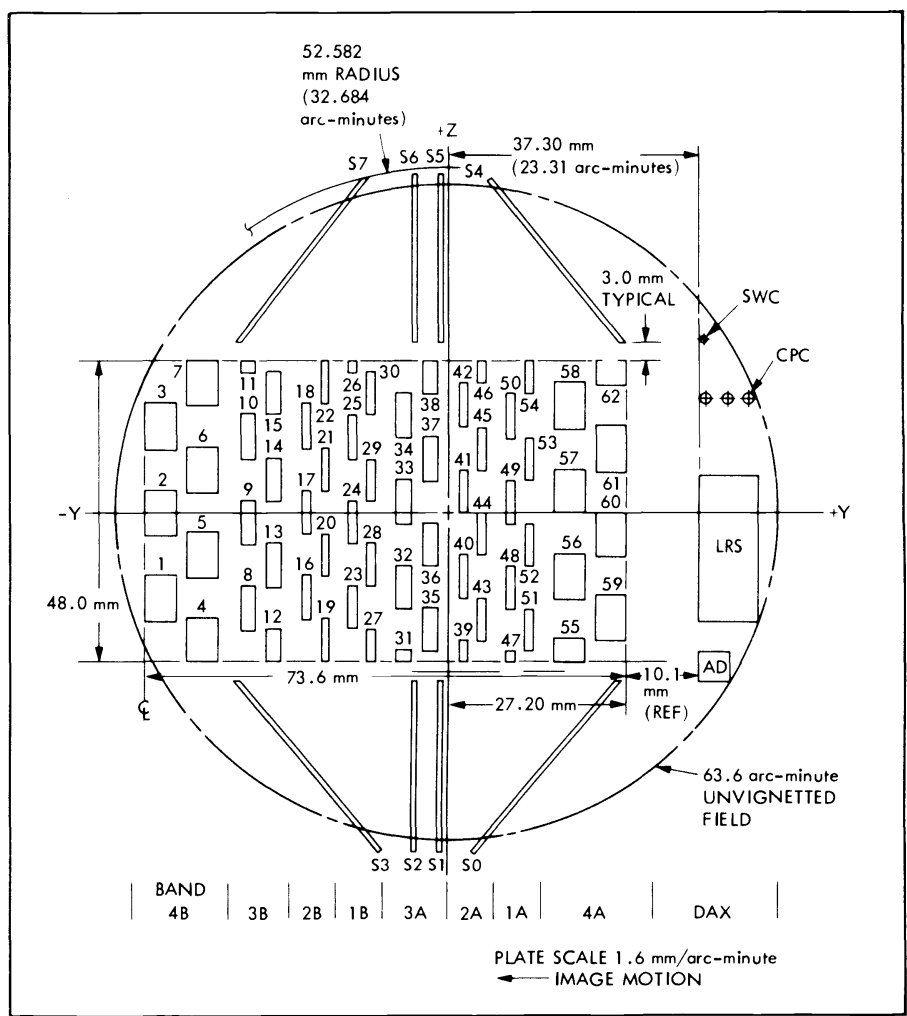

Fig. 3. Layout of focal plane showing detector bands and channel numbers.

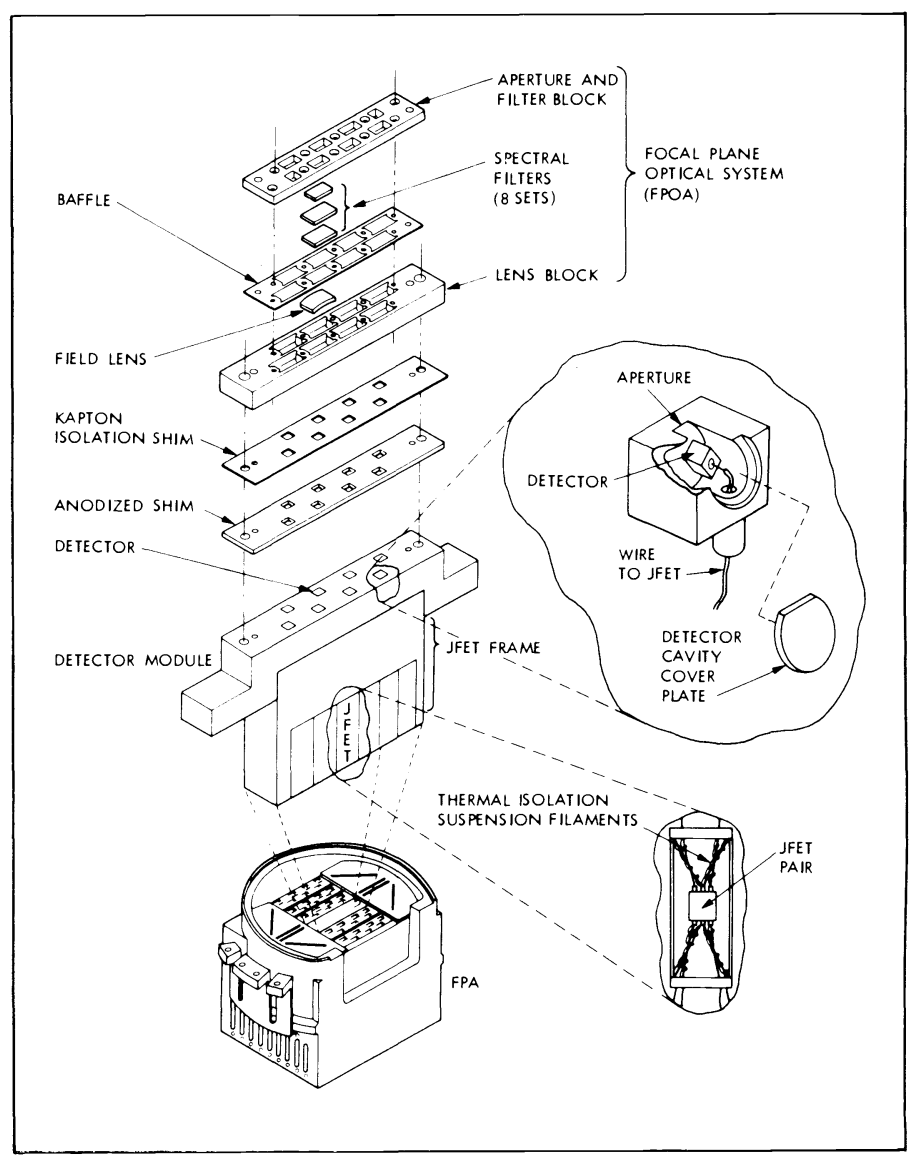

Fig. 4. Detector subarray module showing filters, optics, detectors, and JFET amplifiers. 
TABLE I. Characteristics of IRAS Focal Plane

\begin{tabular}{ll}
\hline Focal plane assembly & \\
Detector operating temperature & $2.8 \mathrm{~K}$ \\
JFET operating temperature & 70 to $80 \mathrm{~K}$ \\
MOSFET operating temperature & $2.8 \mathrm{~K}$ \\
Number of detectors & 62 infrared, 8 visible \\
Power dissipation & $14 \mathrm{~mW}$ \\
Construction & Modular: \\
& 8 infrared subarrays \\
Detector material & 2 visible subarrays \\
Band 1 & \\
Band 2 & $\mathrm{Si}: \mathrm{As}$ \\
Band 3 & $\mathrm{Si}: \mathrm{Sb}$ \\
Band 4 & $\mathrm{Ge}: \mathrm{Ga}$ \\
Visible & $\mathrm{Ge}: \mathrm{Ga}$ \\
& $\mathrm{Si}$ \\
Filter material & \\
Band 1 & $\mathrm{BaF}, \mathrm{Ge}$ \\
Band 2 & $\mathrm{Si}$ \\
Band 3 & $\mathrm{Sapphire}, \mathrm{KRS}-5$ \\
Band 4 & $\mathrm{Sapphire}, \mathrm{C} \mathrm{F}_{2}, \mathrm{KCl}$ \\
Visible & $\mathrm{BG}-38$ \\
& \\
Field lens material & $\mathrm{Ge}$ \\
Band 1 & $\mathrm{Si}$ \\
Band 2 & $\mathrm{Ge}$ \\
Band 3 & $\mathrm{Ge}$ \\
Band 4 & $\mathrm{~N} / \mathrm{A}$ \\
Visible & \\
\hline
\end{tabular}

components in the system that is of great interest to the users of the IRAS and to designers of future systems. Table III gives the results of in-orbit tests under operational conditions.

A comparison of these two sets of test results shows that the in-orbit performance equals or exceeds expected performance based on prelaunch tests. Figure 7 shows scans over the same portion of sky by six different detectors on consecutive orbits. The repeatability of IRAS measurements of weak extended features is obvious. Clearly, astronomers have been provided with an excellent infrared detection system for study of the infrared sky.

\subsection{Ionization radiation effects}

It was found prior to launch but after the design was completed that the infrared detectors used in the focal plane would suffer large changes in noise and responsivity as the result of passage through the intense radiation concentrated in the South Atlantic Anomaly (SAA). The "bias boost" strategy anneals the radiation effects in the detectors by raising the bias voltage so that the detectors "break

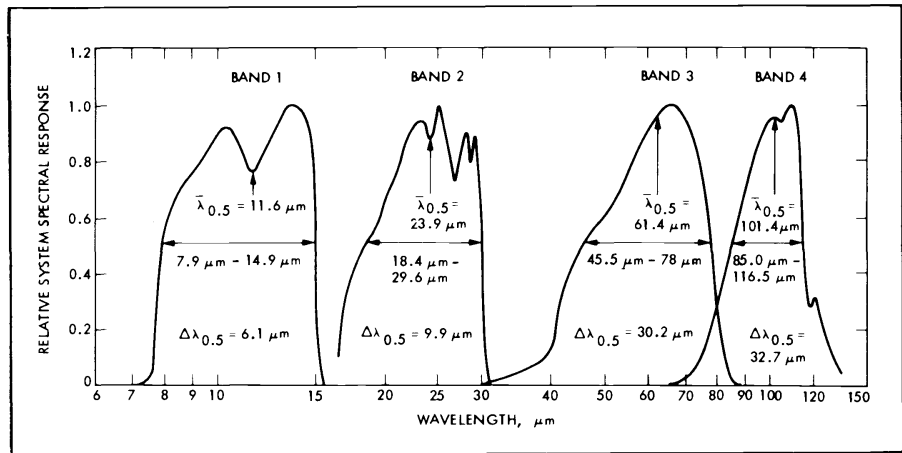

Fig. 5. Relative system spectral response curves for the IR bands.

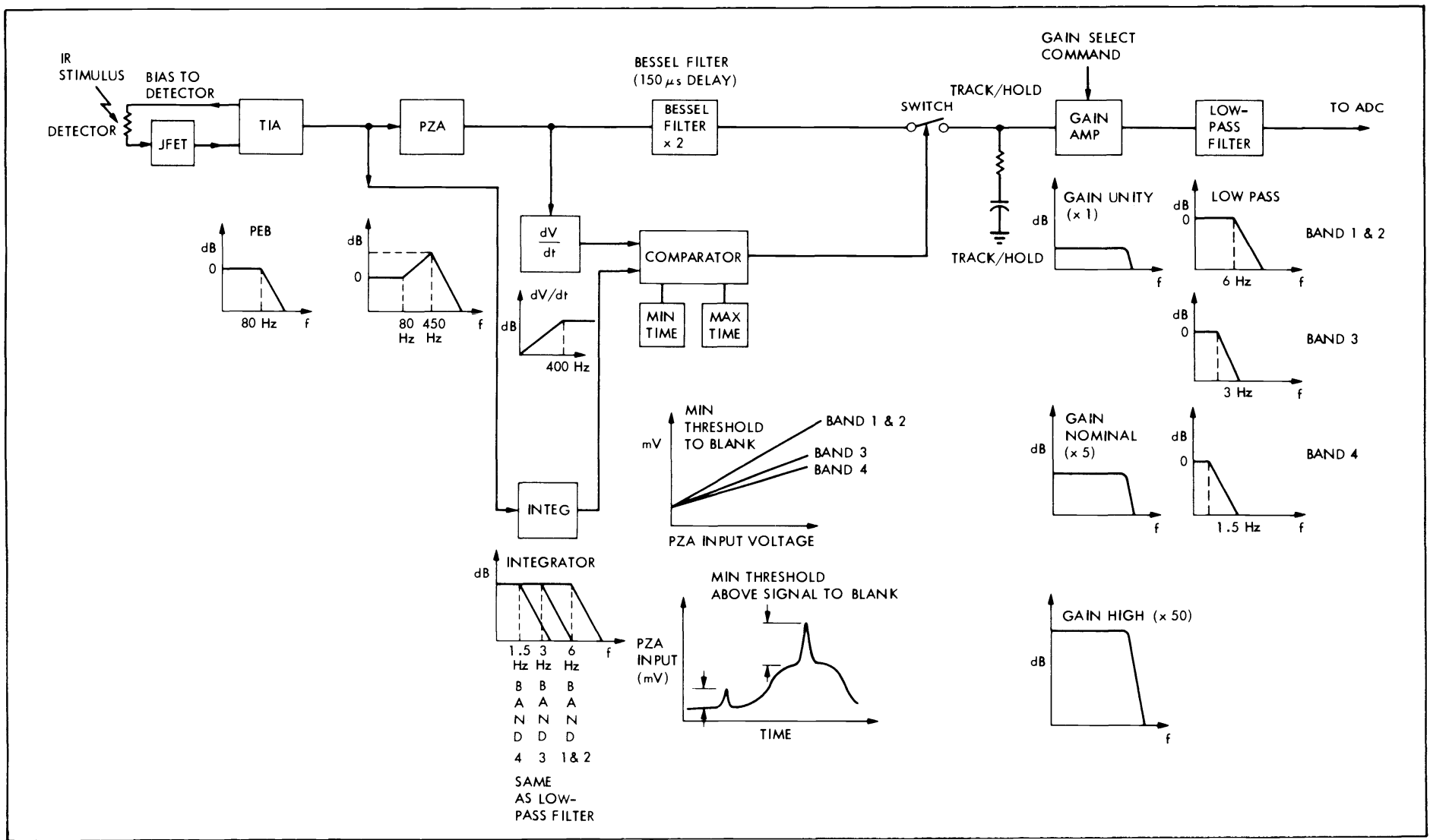

Fig. 6. Infrared data flow. 
Band 1

\section{Spectral response}

Center wavelength

Effective bandwidth

Mean in-band transmission

\section{Out-of-band leaks}

\section{Shortward leak}

Longward leak

\section{Limiting point source sensitivity (NEFD)}

Survey mode $(\mathrm{Jy})^{*}$

Deep sky mode (Jy)

\section{Optical quality}

Diam of $80 \%$ encircled energy

Surface reflectivity

Aperture efficiency

Off-axis source rejection

\section{$A(\theta)$ for Jupiter @ 5 deg}

$A(\theta)$ for moon@24 deg

$A(\theta)$ for sun @60 deg

$A(\theta)$ for earth limb@88 deg

$\begin{array}{lc}11.8 \mu \mathrm{m} & 24.4 \mu \mathrm{m} \\ 5.7 \mu \mathrm{m} & 9.8 \mu \mathrm{m} \\ 0.83 & 0.81\end{array}$

$<1.7 \times 10^{-5}$

$<2.8 \times 10^{-6}$

0.12

0.04

25 arcsec
0.96
0.88

$1.4 \times 10^{0}$

$1.0 \times 10^{-5}$

$5.0 \times 10^{-8}$

$2.0 \times 10^{-8}$

$<4.5 \times 10^{-5}$

$<1.5 \times 10^{-6}$

0.03
Band 2

Band 3

Band 4

$101.2 \mu \mathrm{m}$

$61.6 \mu \mathrm{m}$

$35.6 \mu \mathrm{m}$

0.28

0.15

$\begin{array}{ll}<2.3 \times 10^{-4} & <9.3 \times 10^{-2} \\ <1.5 \times 10^{-4} & <5.4 \times 10^{-2}\end{array}$

0.13

0.30

0.04

0.10

$\begin{array}{cc}50 \text { arcsec } & 100 \text { arcsec } \\ 0.96 & 0.96 \\ 0.87 & 0.92\end{array}$

$<15 \%$

$<15 \%$

$<15 \%$

TABLE III. In-Flight Performance of IRAS Focal Plane

\begin{tabular}{|c|c|c|c|c|c|}
\hline Band & $\begin{array}{l}\text { Optical } \\
\text { efficiency }\end{array}$ & $\begin{array}{c}\text { System } \\
\text { responsivity } \\
(\mathrm{A} / \mathrm{W})\end{array}$ & $\begin{array}{c}\text { Detector } \\
\text { responsivity } \\
(\mathrm{A} / \mathrm{W})\end{array}$ & $\begin{array}{c}\text { NEP } \\
\left(\mathrm{W} / \mathrm{Hz}^{1 / 2}\right)\end{array}$ & $\begin{array}{c}\text { NEFD } \\
\left(\mathrm{Jy} / \mathrm{Hz}^{1 / 2}\right)\end{array}$ \\
\hline 1 & 0.47 & 0.115 & 0.35 & $3 \times 10^{-16}$ & 0.03 \\
\hline 2 & 0.44 & 0.53 & 1.7 & 0.6 & 0.025 \\
\hline 3 & 0.55 & 0.34 & 1.0 & 1.0 & 0.046 \\
\hline 4 & 0.16 & 0.24 & 1.8 & 0.6 & 0.21 \\
\hline
\end{tabular}

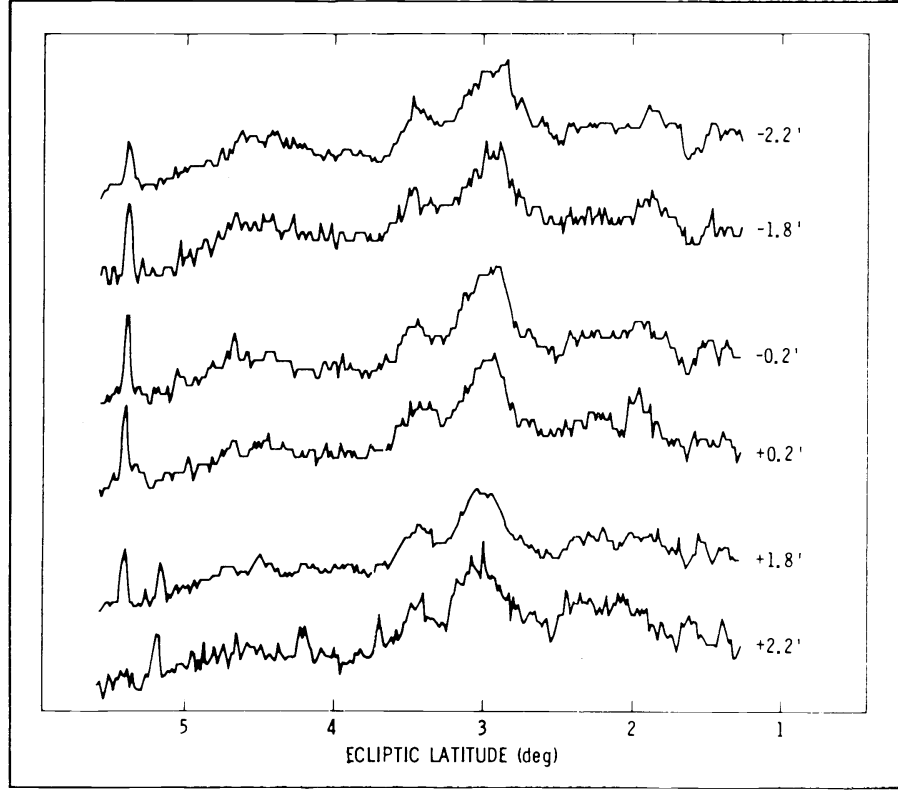

Fig. 7. Scans over portion of sky by six detectors on consecutive orbits.

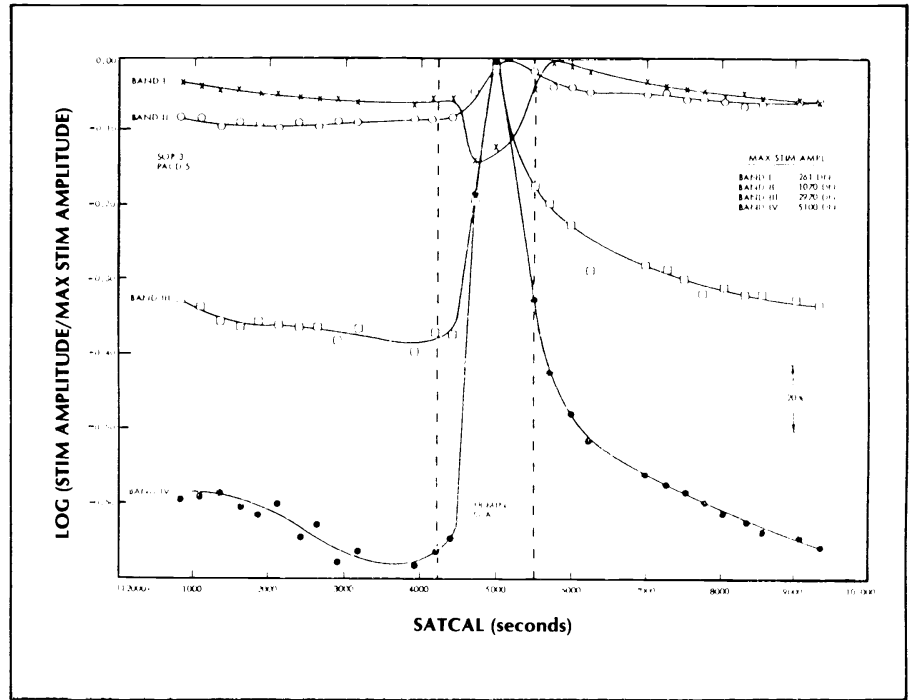

Fig. 8. Large variations in detector responsivity during SAA passages were monitored as a function of time using the output of an infrared calibration source (a "stimulator"). No bias boost was used during this SAA passage.

126 / OPTICAL ENGINEERING / March/April 1984 / Vol. 23 No. 2 


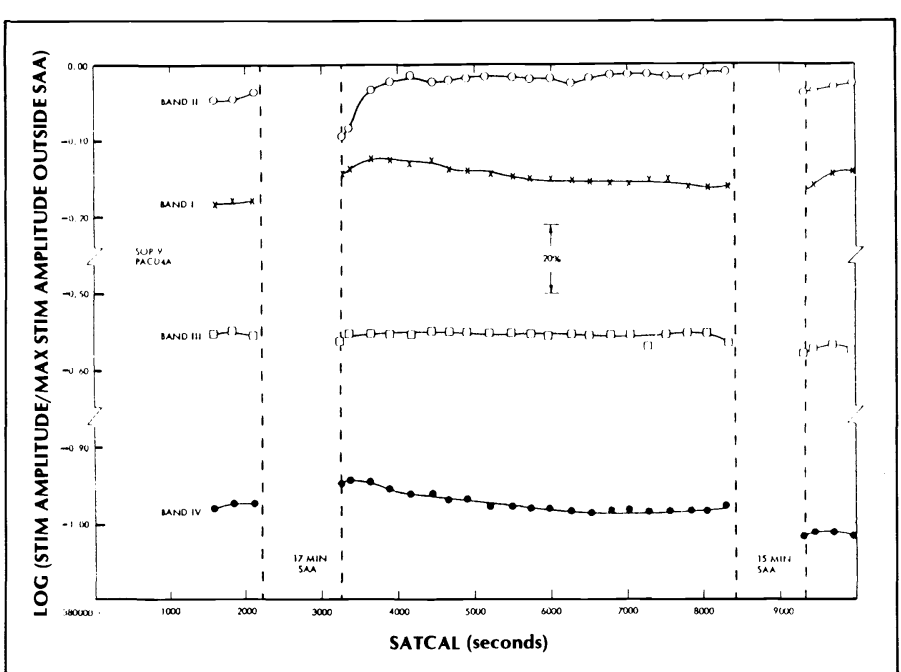

Fig. 9. Monitoring of stimulator amplitude during SAA passages shows little variations in detector responsivity when bias boost is used.

down" during SAA passages. Figures 8 and 9 show in-flight test results on the performance of the bias boost strategy. A dramatic improvement is seen in the behavior of the 60 and $100 \mu \mathrm{m} \mathrm{Ge:Ga}$ detectors.
Other aspects of the particle environment, such as cosmic ray hit rates and performance of the on-board deglitcher, are very much as anticipated.

\section{ACKNOWLEDGMENTS}

The IRAS system performance stands as a lasting tribute to the dedication and skills of its designers and builders.

A portion of the research described in this paper was performed by the Jet Propulsion Laboratory and the California Institute of Technology under contract with the National Aeronautics and Space Administration

\section{REFERENCES}

1. M. O. Harwit, D. P. McNutt, K. Shivanandan, and B. J. Zajac, Appl. Opt. 5, 1732 (1966).

2. M. O. Harwit, J. R. Houck, and K. Fuhrman, Appl. Opt. 8, 473 (1969).

3. B. T. Soifer, J. R. Houck, and M. Harwit, Astrophys. J. 168, L73 (1971).

4. D. P. McNutt, K. Shivanandan, and P. D. Feldman, Appl. Opt. 8, 2199 (1969).

5. D. P. McNutt, K. Shivanandan, M. D. Daehler, and P. D. Feldman, Appl. Opt. 14, 1116(1975)

6. R. G. Walker and S. D. Price, "AFGL Four Color Infrared Sky Survey," AFGL-TR-76-0208 (17 Sept. 1976)

7. C. R. McCreight, R. G. Walker, and F. C. Witteborn, in Utilization of Infrared Detectors, I. J. Spiro, ed., Proc. SPIE 132, 66 (1978).

8. T. Nishimura, F. J. Low, and R. F. Kurtz, Astrophys. J. 239, L101 (1980).

9. W. Irace and D. Rosing, J. Brit. Interplanetary Soc. 36, 27 (1983)

10. F. J. Low, in Infrared Astronomy-Scientific/Military Thrusts \& Instrumentation, N. W. Boggess and H. J. Stears, eds., Proc. SPIE 280, $56(1981)$ 